\title{
Les effets des investissements d'agrobusiness sur les agriculteurs familiaux Le cas de la vallée du fleuve Sénégal
}

\author{
Guillaume SOULLIER • CIRAD, UMR ART-DEV, F-34398 Montpellier, France ; ART-DEV, Univ \\ Montpellier, CIRAD, CNRS, Univ Montpellier 3, Univ Perpignan Via Domitia, Montpellier, France \\ guillaume.soullier@cirad.fr \\ Paule MOUSTIER • CIRAD, UMR MOISA, F-34398 Montpellier, France; MOISA, Univ Montpellier, \\ CIHEAM-IAMM, CIRAD, INRA, Montpellier SupAgro, Montpellier, France \\ moustier@cirad.fr \\ Jérémy BOURGOIN • CIRAD, UMR TETIS, ISRA, Sénégal ; TETIS, Univ Montpellier, AgroParisTech, \\ CIRAD, CNRS, IRSTEA, Montpellier, France \\ jeremy.bourgoin@cirad.fr \\ Alpha BA • ENSA/ Université de Thiès, Département Économie et Sociologie rurale, Sénégal \\ alphaba80@gmail.com
}

Des politiques agricoles en Afrique de l'Ouest soutiennent les investissements d'agrobusiness sur des territoires où vivent des agriculteurs familiaux. Les auteurs étudient les effets de ces investissements sur la gouvernance territoriale, ainsi que sur l'accès au foncier, le revenu, la sécurité alimentaire et les pratiques agricoles des petits producteurs. Le cadre conceptuel est celui de la gouvernance des chaînes de valeur et des territoires. Le territoire étudié est la vallée du fleuve Sénégal, où les auteurs ont réalisé des entretiens semi-directifs, deux enquêtes quantitatives et un atelier participatif. Les résultats montrent que le changement de gouvernance territoriale dépend de la prise en compte des institutions coutumières et légales, et que les effets de la coordination verticale sont différents en fonction des types de producteurs familiaux.

MOTS CLÉS : agriculture familiale, agrobusiness, gouvernance, Afrique, Sénégal

\section{The effects of agribusiness investments on family farmers. The case of the Senegal River Valley}

Agricultural policies in West Africa support investments from agribusiness on territories where family farmers live. The authors study the effects of these investments on the territorial governance, the land access, the income, the food security, and the agricultural practices of small-scale farmers. The conceptual framework is that of the governance of value chains and territories. The territory studied is the Senegal River Valley, where the authors carried out semi-structured interviews, two quantitative surveys, and one participatory workshop. Results show that the change in governance depends on the consideration of customary and legal institutions, and that the effects of vertical coordination are different according to the types of family producers. (JEL: Q13; Q18; Q12; Q15)

KEYWORDS: family farming, agribusiness, governance, Africa, Senegal

$\mathrm{D}$ urant la crise alimentaire de 2007 , l'indice des prix alimentaires de la Banque mondiale a été multiplié par 2,5. En vue d'améliorer la sécurité alimentaire dans les pays en développement, la FAO, la Banque mondiale et plusieurs États africains ont recentré leurs interventions sur les filières domestiques. Ces États, dont le Sénégal, cherchent à devenir « autosuffisants $»$. 
Les politiques publiques cherchent à augmenter la production, en soutenant une agriculture entrepreneuriale. Elles appuient l'aménagement de superficies irriguées et mettent en place des crédits et subventions aux intrants de synthèse (Lançon et Mendez del Villar, 2013). Elles encouragent aussi des entreprises étrangères à réaliser de grands investissements dans la production et la transformation agroalimentaire. Ces entreprises prennent le contrôle hiérarchique de la production ou mettent en place des contrats (Soullier, 2017 ; Hathie, 2016).

Ces investissements sont réalisés sur des territoires où l'agriculture familiale domine. Ils peuvent avoir des effets sur la gestion des ressources agricoles et la performance des petits producteurs. Néanmoins, les politiques et travaux de recherche actuels se concentrent plutôt sur la capacité des filières domestiques à satisfaire les besoins alimentaires des consommateurs urbains (Hathie, 2016). Nous cherchons dans cet article à contribuer à la compréhension de deux phénomènes : (1) Comment des investissements d'agrobusiness (AB) dans la production et la transformation influencent-ils la participation des petits producteurs à la gestion des ressources agricoles du territoire ? (2) Quels sont les effets des changements de l'organisation des filières et des territoires sur la performance des petits producteurs ? Nous proposons de répondre à ces questions par l'étude d'investissements dans le delta de la vallée du fleuve Sénégal, où le changement d'organisation des filières semble affecter les petits producteurs en termes d'accès au foncier, de revenus, de sécurité alimentaire et de pratiques agricoles (Soullier et Moustier, 2015, 2018).

\section{Cadre conceptuel : gouvernance des chaînes de valeur et gouvernance territoriale}

Les agriculteurs familiaux ${ }^{1}$ sont caractérisés par la gestion familiale du capital, du travail et de la production, ainsi que la présence d'autoconsommation et un statut juridique parfois informel. Ils peuvent participer à une chaîne de valeur traditionnelle, qui est composée de plusieurs intermédiaires faiblement dotés en capital, ou à une chaîne de valeur moderne, qui comprend des $\mathrm{AB}$. Ces $\mathrm{AB}$ sont des entreprises privées, intégrées aux marchés locaux et/ou internationaux, qui mettent en œuvre une agriculture intensive et/ou des activités de transformation basées sur des moyens de production à fort contenu en capital.

La définition d'une chaîne de valeur est proche de celle d'une filière. La filière regroupe l'ensemble des activités de production, de transformation et d'acheminement qui sont nécessaires à la fourniture d'un bien ou d'un service au consommateur final. Le cadre conceptuel de la gouvernance des chaînes de valeur permet d'expliquer les formes d'organisation plus ou moins intégrées (c'est-à-dire combinant plusieurs activités de la chaîne, par exemple, production et transformation), et leurs effets économiques sur les producteurs (Gereffi et al., 2005). Il établit le lien entre le pilotage de la qualité du produit par la firme dite dominante ainsi que la distribution des compétences et de la valeur ajoutée entre les acteurs. L'upgrading, qui peut être traduit par " mise à niveau », est le processus d'acquisition de nouvelles capacités et d'accès à de nouveaux marchés par la participation à une chaîne

1. Dans cet article, nous utilisons indifféremment les termes « petit producteur» et « agriculteur familial » car les agriculteurs familiaux sont caractérisés par une petite taille d'exploitation (moins de $5 \mathrm{ha}$ ). 
de valeur (Humphrey, 2004). Il peut être provoqué par l'utilisation de nouvelles technologies et combiné avec de nouvelles formes de gouvernance. La gouvernance est définie comme les « relations d'autorité et de pouvoir qui déterminent comment les ressources financières, matérielles et humaines sont réparties et circulent au sein de la chaîne » (Gereffi et Korzeniewicz, 1994) ${ }^{2}$. La gouvernance varie entre le marché (aussi appelé transaction spot), où la coordination est réalisée par le prix, et la hiérarchie, où l'approvisionnement est contrôlé par la voie administrative. Des formes intermédiaires de gouvernance concernent les contrats.

La performance des producteurs dépend non seulement de l'organisation des chaînes de valeur, mais aussi de leurs accès aux ressources agricoles des territoires. Cet accès est influencé par les investissements des $\mathrm{AB}$, qui peuvent être soutenus par les autorités nationales et locales. La gouvernance territoriale est un " processus dynamique de coordination entre des acteurs publics et privés aux identités multiples et aux ressources asymétriques autour d'enjeux territorialisés » (Rey-Valette et al., 2011). La gouvernance territoriale permet de saisir la diversité des acteurs autour d'enjeux au sein d'un espace déterminé. Les formes de coordination territoriale caractérisent différents niveaux de participation des groupes d'acteurs (Beuret, 2006). Elles varient entre la communication, lorsque des acteurs font passer un message concernant une décision, et la négociation, lorsqu'il y a construction d'une décision commune. Le conflit est un moyen pour des parties écartées de la négociation de réintégrer le processus (Chia et al., 2008). Ce cadre est dynamique puisque des innovations technologiques, organisationnelles et institutionnelles peuvent changer ce niveau de

2. Traduction des auteurs. participation, notamment lorsqu'elles ont lieu au sein d'une chaîne de valeur.

\section{Hypothèses et chemins d'impacts}

La première hypothèse est que les $A B$ sont caractérisés par de la coordination verticale et une grande échelle de production, résultant en une forte pression sur la gouvernance territoriale. L'installation d'AB conduirait donc à une réduction de la participation des petits producteurs dans la gestion des ressources agricoles du territoire (Amanor, 2012).

La seconde hypothèse est que ces investissements provoquent des effets dont les aspects positifs et négatifs varient en fonction des types de producteurs (Medernach et Burnod, 2013). Nous analysons dans cet article les effets sur l'accès au foncier, les revenus, la sécurité alimentaire et les pratiques agricoles (figure 1). La littérature documente les effets significativement positifs et négatifs des investissements sur ces variables (figure 1).

- Les investissements à grande échelle réduisent l'accès au foncier et à l'eau des producteurs familiaux (Amanor, 2012 ; Burnod et al., 2012).

- Les effets de la coordination verticale sur les revenus des producteurs familiaux sont encore incertains. Une partie de la littérature documente des effets positifs. Les contrats provoquent l'upgrading des petits producteurs inclus, en leur permettant d'accéder à des intrants améliorés et à des conseils techniques (Reardon et al., 2009). De plus, le contrôle hiérarchique de la production agricole améliore les revenus des petits producteurs par le biais du marché du travail, notamment pour les plus pauvres (Van den Broeck et al., 2017). Néanmoins, d'autres études montrent l'absence d'effet des contrats sur les revenus car les producteurs ont une capacité de négociation limitée. 


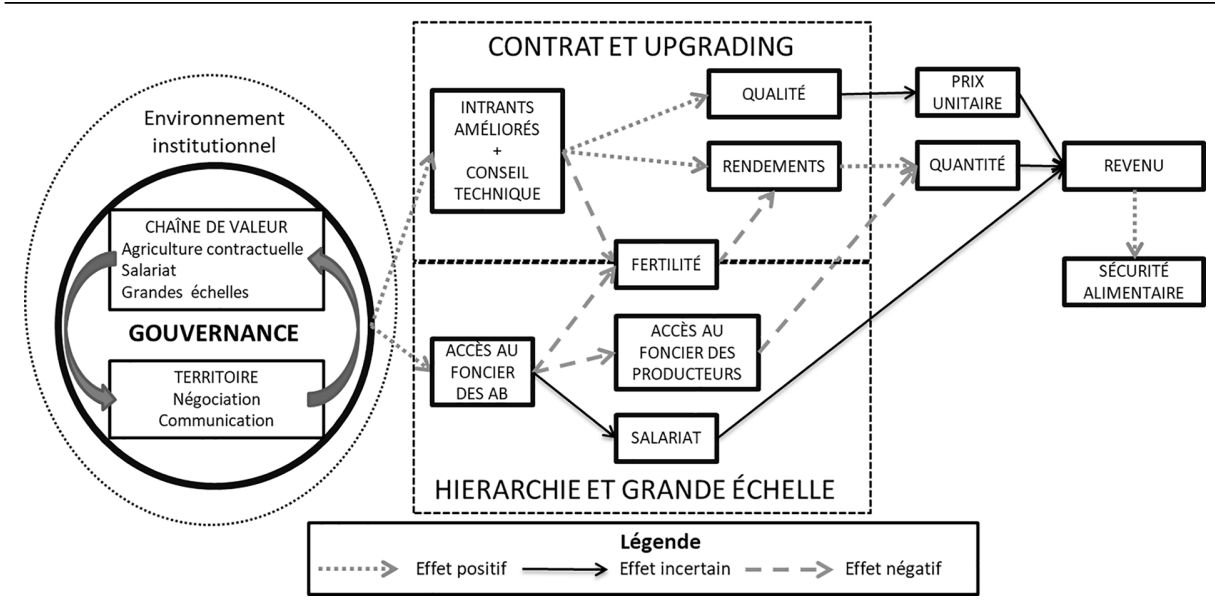

Source : revue de la littérature citée.

De plus, les emplois auprès d'AB sont délaissés par les producteurs locaux, et occupés par des migrants (Burnod et al., 2012).

- L'agriculture contractuelle a un effet positif sur la sécurité alimentaire lorsqu'elle a un effet positif sur le revenu (Bellemare et Novak, 2017). Elle ne se substitue pas aux cultures vivrières (Burnod et al., 2012).

- Ces effets positifs pourraient ne pas durer dans le temps puisque l'intensification de la production agricole peut aboutir à la réduction de la fertilité des sols et des rendements, en particulier lorsqu'une entreprise recherche une rentabilité de court terme (Opondo, 2000).

De plus, les investissements d'AB ont des effets qui varient en fonction des types de producteurs. Par exemple, des investissements dans la production d'agrocarburants à Madagascar ont des effets négatifs sur les revenus et sur l'accès au foncier des grands agriculteurs et des éleveurs, mais positifs pour les petits producteurs et les migrants, notamment par le biais du salariat (Medernach et Burnod, 2013).

\section{Méthode. Une approche mixte}

Le terrain d'étude est le delta du fleuve Sénégal, où trois chaînes de valeur sont concernées par des investissements d'AB : la chaîne de valeur du riz (Soullier et Moustier, 2015), la chaîne de valeur des agrocarburants (Actionaid, 2014) et la chaîne de valeur des produits maraîchers, en particulier de la tomate et de l'oignon (Van den Broeck et al., 2017). Nous utilisons une approche mixte. Nous avons réalisé 154 entretiens semi-directifs avec des acteurs des chaînes de valeur et des agents de développement et de la recherche. Les questions abordées étaient l'organisation des chaînes de valeur et $\mathrm{du}$ territoire, les politiques publiques, les comportements d'acteurs, la gestion de la technologie et la qualité des produits.

Trois études de cas d'AB récemment implantés ont été réalisées. Les $\mathrm{AB}$ ont été sélectionnés de manière raisonnée, sur les critères de la diversité des formes de coordination avec les producteurs (contrats et salariat) et leur localisation (présentée par la figure 2). Puisqu'une étude des effets des $\mathrm{AB}$ sur les revenus des producteurs était en cours dans la commune de Diama (Van 
den Broeck et al., 2017), nous avons sélectionné des $\mathrm{AB}$ dans d'autres communes. L'analyse de l'insertion territoriale des AB se fait pour des espaces couvrant une partie du delta du fleuve Sénégal. Elle intègre l'échelon villageois, où se déroulent les activités agricoles, et l'échelon communal, où l'affectation officielle des parcelles à exploiter est faite par la collectivité locale. $\mathrm{Au}$ total, 332 petits producteurs situés à proximité des $\mathrm{AB}$ ont été aléatoirement sélectionnés et enquêtés ${ }^{3}$. Cette enquête réalisée en 2015 traite des caractéristiques des ménages de producteurs, de leurs activités économiques, de leurs liens avec les $\mathrm{AB}$ (en termes de foncier, salariat et transactions), de leurs pratiques agricoles et de leur sécurité alimentaire.

Un atelier participatif a été réalisé afin de discuter des modalités de négociations formelles et informelles ainsi que des compromis liés à l'installation et l'extension d'AB. Cet atelier a regroupé des représentants d'AB, d'agriculteurs et d'éleveurs autour d'une activité de simulation participative de négociation foncière. La simulation est basée sur le jeu de rôle TerriStoriesTM ${ }^{4}$, développé pour discuter des usages et des pratiques foncières dans le contexte sahélien. Ce type d'atelier permet de discuter les résultats d'enquêtes et fournit l'opportunité pour chaque acteur de comprendre les logiques des autres acteurs et de les discuter.

Une seconde enquête concerne les pratiques agricoles. Quelques 118 parcelles de riz ont été suivies durant la contre-saison 2016. Ces parcelles ont été sélectionnées aléatoirement à partir de

3. Le détail est de 100 producteurs pour l'étude du cas de l'AB Coumba Nor Thiam, 133 pour l'AB West African Farm et 99 pour l'AB Senhuile-Senethanol.

4. http://www.terristories.org/fr/index.html. Il s'agit d'un jeu de rôle créé par Patrick d'Aquino (Cirad). listes fournies par la Société d'aménagement et d'exploitation des terres du delta du fleuve Sénégal, des vallées du fleuve Sénégal et de la Falémé (SAED), et par les riziers. Quelques 27 parcelles cultivées dans le cadre de contrats de production avec un rizier et 30 parcelles gérées hiérarchiquement par des transformateurs de paddy sont comparées avec 61 parcelles cultivées par des producteurs commercialisant le paddy par des transactions spot. La durabilité des pratiques agricoles est comprise comme la reproduction de la fertilité des sols. Les indicateurs sont la nature et les quantités des intrants utilisés, la fréquence des labours, l'intensité culturale et les modalités de l'irrigation.

Nous présentons aussi les résultats de recherches réalisées afin d'estimer l'impact de contrats sur les revenus et l'insécurité alimentaire des producteurs (Soullier et Moustier, 2018). En tout, $607^{5}$ producteurs ont été sélectionnés aléatoirement à partir de la liste des organisations de producteurs ayant cultivé en contre-saison 2014. Les indicateurs de revenu sont le prix de vente et la marge nette ${ }^{6}$ par kilogramme de paddy commercialisé. L'indicateur d'insécurité alimentaire est le Household Food Insecurity Access Scale, qui mesure la perception des répondants quant à la dimension accès de la sécurité alimentaire? ${ }^{7}$ Les impacts de la coordination verticale sont estimés en corrigeant le biais de sélection avec un modèle à

5. Le détail est de 265 producteurs vendant par des transactions spot, 130 producteurs vendant par contrat de commercialisation, 155 producteurs vendant par contrat de production et 44 n'ayant pas cultivé de riz du fait de l'absence de crédit.

6. Nous déduisons aussi l'amortissement du capital et les coûts d'opportunité.

7. Neuf questions appréhendent cette dimension. Le répondant dispose de trois options de fréquence. Le score varie entre 0 et 27. 
Tableau 1. Méthodes, échantillons et indicateurs de la collecte de données

\begin{tabular}{|c|c|c|c|c|c|c|}
\hline & \multicolumn{2}{|c|}{$\begin{array}{c}\text { Gouvernance des chaînes } \\
\text { de valeur et des } \\
\text { territoires }\end{array}$} & Accès au foncier & \multirow{2}{*}{\multicolumn{2}{|c|}{\begin{tabular}{c|c} 
Revenu & $\begin{array}{c}\text { Insécurité } \\
\text { alimentaire }\end{array}$ \\
$\begin{array}{c}\text { Enquête et évaluation } \\
\text { d'impact }\end{array}$
\end{tabular}}} & \multirow{2}{*}{$\begin{array}{l}\begin{array}{c}\text { Pratiques } \\
\text { agricoles }\end{array} \\
\text { Enquête et } \\
\text { statistiques } \\
\text { descriptives }\end{array}$} \\
\hline Méthodes & $\begin{array}{c}\text { Entretiens } \\
\text { semi- } \\
\text { structurés }\end{array}$ & $\begin{array}{l}\text { Études de cas par } \\
\text { enquête }\end{array}$ & $\begin{array}{c}1 \text { atelier } \\
\text { participatif }\end{array}$ & & & \\
\hline Échantillons & 154 entretiens & $\begin{array}{c}3 \mathrm{AB} \\
332 \text { petits } \\
\text { producteurs }\end{array}$ & $\begin{array}{c}\text { Panel multi- } \\
\text { acteurs de } \\
34 \\
\text { personnes }\end{array}$ & \multicolumn{2}{|c|}{$\begin{array}{l}\text { Enquête auprès de } 607 \\
\text { producteurs }\end{array}$} & $\begin{array}{l}\text { Suivi de la } \\
\text { culture de } 118 \\
\text { parcelles }\end{array}$ \\
\hline Indicateurs & $\begin{array}{l}\text { Politiques } \\
\text { publiques, } \\
\text { coordination } \\
\text { des acteurs, } \\
\text { technologies, } \\
\text { qualité des } \\
\text { produits }\end{array}$ & $\begin{array}{l}\text { Caractéristiques } \\
\text { des ménages, } \\
\text { activités } \\
\text { économiques, } \\
\text { liens avec les AB, } \\
\text { pratiques } \\
\text { agricoles et } \\
\text { insécurité } \\
\text { alimentaire }\end{array}$ & $\begin{array}{c}\text { Propositions } \\
\text { / Demandes } \\
\text { de } \\
\text { contreparties } \\
\text { /compensa- } \\
\text { tions }\end{array}$ & $\begin{array}{l}\text { Prix de } \\
\text { vente et } \\
\text { marge } \\
\text { nette par } \\
\text { kg }\end{array}$ & $\begin{array}{l}\text { Household } \\
\text { Food } \\
\text { Insecurity } \\
\text { Access Scale }\end{array}$ & $\begin{array}{c}\text { Nature et } \\
\text { quantités des } \\
\text { intrants, } \\
\text { fréquence des } \\
\text { labours, intensité } \\
\text { culturale et } \\
\text { modalités de } \\
\text { l'irrigation. }\end{array}$ \\
\hline
\end{tabular}

Source : les auteurs.

variable instrumentale ${ }^{8}$ et un modèle d'appariement au score de propension?

\section{Résultats}

\section{Avant la crise des prix de 2007}

\section{Gouvernance des chaînes de valeur}

Entre la mise en œuvre des ajustements structurels (1987) et la crise des prix (2007), les riziculteurs commercialisaient le paddy par des transactions spot, incluant une proximité relationnelle avec l'acheteur (Soullier et Moustier, 2015). La technique utilisée ne réalisait que le décorticage, ce qui produisait un riz comportant des impuretés. Les producteurs d'oignons étaient impliqués dans une chaîne de valeur dans laquelle se posaient aussi des enjeux de qualité (Diouf, 2014). Par contre, la chaîne de valeur de la tomate

8. Les instruments sont la distance au rizier le plus proche proposant un contrat de production et la perception des producteurs quant à l'incertitude portant sur leur capacité à financer à crédit la culture du riz.

9. La validité des hypothèses d'indépendance conditionnelle et de support commun est vérifiée. était caractérisée par l'existence d'un AB, créé en 1965 (Diouf, 2016).

\section{Gouvernance territoriale}

Dans le delta, les principales ressources relatives à l'agriculture sont le foncier agricole, les espaces de pâturage, l'eau servant à l'irrigation et à l'abreuvement $\mathrm{du}$ bétail, et les ressources naturelles végétales. Le département comprend trois zones agroécologiques (figure 2). Le Walo est composé de terres humides proches du fleuve, favorables aux cultures irriguées sur des sols sableux (fonde), argileux (hollalde) et intermédiaires (faux hollalde). Le Diéri est traversé par le lac de Guiers et comprend des sols sablonneux éloignés du fleuve. Le Ferlo est la zone la plus aride du territoire.

Les principales ethnies vivant sur le territoire sont les Wolofs, les Maures et les Peulhs. Bien que la segmentation spatiale ne soit pas absolue, les zones agroécologiques influencent la localisation des activités agricoles et des ethnies. Les producteurs spécialisés dans la production de riz sont principalement localisés dans le Walo, ceux réalisant des cultures pluviales 


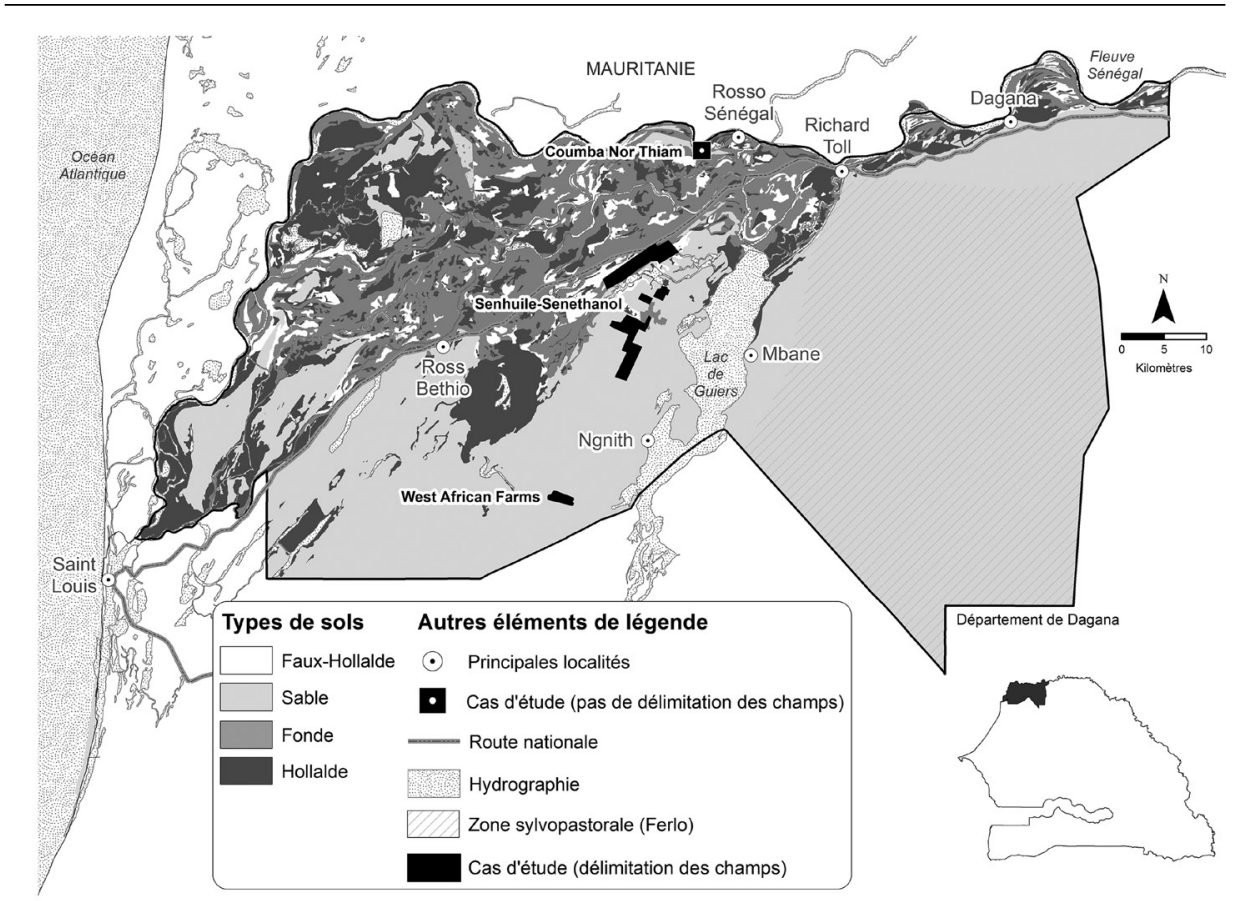

Source : les auteurs.

dans le Diéri, et les maraîchers sont surtout localisés sur les berges du lac de Guiers et du fleuve Sénégal. Ces cultures sont réalisées par des membres des trois ethnies, avec une majorité de Wolofs et de Maures. Les agropasteurs sont des Peulhs, qui sont en grande majorité localisés dans le Ferlo.

Des institutions coutumières et légales régissent l'utilisation des ressources agricoles (Kamara, 2014). D'une part, l'organisation coutumière fait la promotion d'une propriété collective, indivisible et inaliénable, où la descendance hérite du droit d'usage. Elle est caractérisée par une régulation des différentes activités agricoles dans l'espace et le temps. Des complémentarités existent entre les cultures et l'élevage. Par exemple, après la récolte, les éleveurs ont accès aux champs, ce qui permet une alimentation pour le bétail et l'apport d'engrais naturels pour les cultures. Cette gestion coutumière permet de limiter la compétition dans l'utilisation des ressources et donc de limiter les conflits entre les différents types d'agriculteurs. De plus, les différents espaces du territoire ont une valeur symbolique forte pour les agriculteurs familiaux. En effet, leurs activités agricoles, culturelles et religieuses, ont façonné depuis des décennies l'organisation des espaces. L'appropriation du territoire qui en découle en fait un composant fort de l'identité des agriculteurs (Kamara, 2014).

D'autre part, une institution légale régit les espaces. La loi du domaine national classe sous zones de terroirs les terres qui sont utilisées pour l'habitat rural, la culture ou l'élevage, et sous zones classées les réserves écologiques et forestières. Le conseil municipal des collectivités 
locales ou communes ${ }^{10}$ gère l'affectation des zones de terroirs, sous l'autorité de l'État. Il définit le plan d'occupation du sol et est consulté à propos des projets de développement, d'aménagement ou de mise en valeur du terroir. Il délibère sur les demandes d'affectations foncières, quels que soient les types d'acteurs (AB ou agriculteurs familiaux). L'État gère les zones classées.

Le delta est caractérisé par le développement d'aménagements hydro-agricoles et une forte volonté étatique d'intensifier la production agricole. Depuis l'indépendance du pays, la SAED met en œuvre la politique agricole dans la vallée du fleuve Sénégal. Elle appuie depuis 1973 les agriculteurs dans le développement d'une agriculture intensive. Depuis la mise en œuvre des plans d'ajustements structurels à partir de 1987, la SAED s'est désengagée de l'accompagnement au développement rural, et son rôle s'est progressivement limité au conseil agricole, à la gestion des espaces aménagés, ainsi qu'à l'appui aux collectivités locales pour la gestion foncière. Cela a favorisé, par ailleurs, le développement de périmètres privés aménagés par des promoteurs individuels ou organisés en groupements d'intérêt économique.

La Caisse nationale du Crédit Agricole du Sénégal (CNCAS) est une banque agricole avec participation de l'État qui appuie depuis 1987 le développement de l'agriculture par l'offre de crédit. Les producteurs sont rassemblés en organisations de producteurs pour accéder au crédit, aux intrants et réaliser des ventes servant au remboursement du crédit. Néanmoins, certaines organisations de producteurs ont été exclues de l'accès au crédit de la CNCAS pour cause de défaut de remboursement (Fall, 2006). Les raisons de ce non-remboursement incluent une faible

10. Depuis l'acte 3 de la décentralisation (loi $\mathrm{n}^{\circ}$ 2013-10 du 28 décembre 2013). production du fait de chocs climatiques, et l'opportunisme de certains producteurs. Cette situation a favorisé l'arrivée de riziers, qui offrent à crédit des intrants aux petits producteurs.

Les organisations de la société civile sont de deux types. D'une part, des organisations faitières au niveau national ont des cellules locales. Il s'agit par exemple $\mathrm{du}$ Conseil national de concertation et de coopération des ruraux (CNCR) et de la Fédération des organisations non gouvernementales du Sénégal (FONGS). D'autre part, certaines organisations sont locales, comme l'Association socio-éducative, sportive et culturelle de l'Amicale du Walo (ASESCAW). Ces associations regroupent notamment différents types de producteurs.

La gouvernance territoriale du delta est complexe. Elle intègre divers acteurs autour de l'usage des ressources agricoles. L'expansion des surfaces irriguées peut être source de conflits. Certaines collectivités locales s'inspirent des règles coutumières pour asseoir les règles légales. Ainsi, pour l'installation de l'agriculture irriguée, la SAED et les autorités étatiques et locales se sont appuyées sur les droits fonciers locaux des cultivateurs. Néanmoins, la mise en œuvre des règles légales n'est pas toujours compatible avec les règles coutumières. En effet, les institutions coutumières et légales peuvent attribuer des fonctions antagonistes à un même espace. L'expansion des zones irriguées a historiquement pu être source de conflits entre cultivateurs et agropasteurs. En effet, l'évolution du cadre légal a appuyé le développement de cultures sur des espaces traditionnellement dévolus au pâturage transhumant (Kamara, 2014).

\section{Après la crise des prix (depuis 2008)}

\section{Changement de politiques publiques}

Depuis la mise en œuvre des ajustements structurels, les politiques publiques ont 
favorisé des approvisionnements alimentaires à partir des marchés internationaux, en particulier pour le riz. Mais à la suite de la crise alimentaire de 2007, le Gouvernement du Sénégal a mis en place la Grande offensive agricole pour la nourriture et l'abondance (GOANA), qui vise à augmenter la production agricole. Les leviers techniques de l'intensification sont les engrais de synthèse, les semences améliorées, la mécanisation agricole et l'aménagement de surfaces irriguées. Ces mesures sont accompagnées par la mise en place de crédits de campagne et de subventions pour les engrais. Le lancement de cette politique a favorisé un accroissement des affectations de terres à l'élite politique et intellectuelle du Sénégal. Les investissements étrangers sont aussi encouragés, par le biais de l'agence chargée de la promotion de l'investissement et des grands travaux (APIX). Le programme d'accélération de la cadence de l'agriculture sénégalaise encourage la coordination entre les $\mathrm{AB}$ et les agriculteurs familiaux (D'Aquino et Seck, 2013). Le riz est la principale cible de ces politiques (MA, 2009). Sont aussi concernés l'oignon, l'arachide et les fruits et légumes. Enfin, le Projet de développement inclusif et durable de l'agrobusiness au Sénégal (PDIDAS) est financé par le Gouvernement avec un crédit de 40 milliards d'euros de la Banque mondiale. Entre 2014 et 2020, il vise à sécuriser et promouvoir les investissements d'AB dans le delta, notamment par le biais d'investissements publics dans les infrastructures (irrigation et stockage).

\section{Changement de gouvernance des chaînes de valeur}

La chaine de valeur du riz rencontrait des difficultés de qualité du produit et de remboursement des crédits des producteurs à la CNCAS (Fall, 2006). Entre 2010 et 2014, huit transformateurs utilisant des techniques réalisant des fonctions telles que le séchage et le tri sont apparus (Soullier et Moustier, 2015). Ils mobilisent des formes intégrées de coordination avec les producteurs. Les politiques publiques ont appuyé la mise en place de contrats de commercialisation, intégrant les critères de qualité du paddy (taux d'humidité et d'impureté). Les producteurs fournissent un volume correspondant au montant de leur crédit, et les riziers payent directement la banque. Des riziers ont aussi mis en place des contrats de production, dans lesquels ils apportent des intrants et conseils techniques aux producteurs, et sont remboursés en paddy. Chaque type de contrat représentait environ $5 \%$ des volumes produits durant la contre-saison 2014. De plus, quatre rizeries contrôlaient hiérarchiquement la culture de 1500 ha, soit $2 \%$ de la production.

La chaîne de valeur domestique de la tomate rassemblait en 2015 trois entreprises industrielles de transformation s'approvisionnant par le biais de contrats (Diouf, 2016). Cette chaîne de valeur rencontre des difficultés face aux importations de triple concentré de tomate. En parallèle, il y a eu une croissance des investissements dans la production horticole dans le but d'exporter vers les marchés européens (Van den Broeck et al., 2017). Cinq entreprises de capitaux étrangers sont apparues à partir de 2005 dans la commune de Diama. Elles produisent de la tomate, des haricots et des mangues. Elles emploient 5000 personnes dans les champs et unités de conditionnement.

L'entreprise Senhuile-Senethanol a investi dans la production à grande échelle mais la nature de ses cultures reste incertaine (Actionaid, 2014). L'objectif premièrement annoncé était la production de tournesols devant être transformés en agrocarburants. Néanmoins, l'entreprise oriente progressivement ses activités vers la production de maïs et de riz. 


\section{Changement de la gouvernance territoriale}

L'étude de trois AB (Soullier et al., 2016) révèle des insertions territoriales différentes (tableau 2). L'entreprise Coumba Nor Thiam fut créée en 1987 dans le nord du département, ou sont localisés des producteurs spécialisés dans le riz. Elle a progressivement investi dans la production, la mécanisation agricole et la transformation, parfois avec l'appui d'agences de développement. En 2014, elle a cultivé 1000 hectares, réalisé des contrats de production avec 660 producteurs et a transformé 9600 tonnes de paddy. En tout, $76 \%$ des exploitations localisées dans le même village que l'entreprise ont réalisé un contrat de production avec celle-ci, et $2 \%$ ont un membre du ménage qui travaille pour l'entreprise en tant que salarié. L'accroissement des surfaces directement cultivées et contractualisées a permis à l'entreprise d'augmenter son influence sur le foncier. Elle dispose aussi d'une station de pompage.

West African Farms s'est implantée en 2011 dans la commune de Ngnith, où sont localisés des producteurs diversifiés. Le contrat d'exploitation signé avec les représentants de la commune rurale de Ngnith et de l'arrondissement de Ndiaye prévoyait la mise à disposition de 200 ha pour que l'entreprise réalise du maraîchage. En contrepartie, cette dernière devait prioriser des embauches dans la commune, contribuer à son budget, et construire un canal permettant l'irrigation de 200 ha pour les producteurs (Commune rurale de Ngnith, 2011). En 2015, 9,02 \% des producteurs enquêtés travaillaient comme salariés pour l'entreprise. L'aménagement des parcelles, qui devait être fait par les producteurs, n'avait été réalisé que sur 100 ha. En effet, les règles d'attribution des parcelles aux producteurs n'avaient pas été précisées dans le contrat d'exploitation.
L'entreprise ne s'approvisionne pas auprès de producteurs familiaux.

Senhuile-Senethanol est une entreprise italo-sénégalaise qui a été en conflit à propos de son implantation avec la population de la commune de Fanaye, en amont dans la vallée du fleuve Sénégal. Deux décrets présidentiels lui ont ensuite attribué 20000 hectares de la réserve naturelle de Ndiael, à l'ouest du lac de Guiers (Actionaid, 2014). Les cultures de l'entreprise rentrent en conflit avec l'élevage et la culture non irriguée de 9000 agropasteurs, qui réalisent leurs activités dans la réserve depuis plusieurs décennies. Ils utilisent coutumièrement des terres non immatriculées, où il est illégal de cultiver selon la loi foncière nationale (Kamara, 2014). Certains d'entre eux (16,16\%) rapportent l'accaparement de terres qu'ils cultivaient avant l'arrivée de l'entreprise. De plus, l'espace occupé par l'entreprise obstrue les parcours pastoraux qui permettaient aux éleveurs d'accéder aux eaux du lac de Guiers et du fleuve Sénégal. Les 37 villages affectés par les cultures de Senhuile-Senethanol se sont regroupés pour s'opposer à cette occupation. Ils ont formé le « Collectif pour la sauvegarde des terres du Ndiael », dans le but de communiquer auprès des citoyens et d'assurer un suivi de l'occupation des terres (Collectif de Ndiaël et al., 2014). L'entreprise continue l'extension des surfaces cultivées (Actionaid, 2014) bien qu'une décision du gouverneur ait réduit l'affectation des terres à 10000 ha.

Ces trois cas présentent des formes différentes de gouvernance territoriale entre les $\mathrm{AB}$ et les petits producteurs. L'entreprise Coumba Nor Thiam est issue du territoire. Elle connaît les institutions coutumières et dispose d'une proximité avec les producteurs. Parfois avec le soutien d'organisations de développement, elle accroît progressivement son influence sur le foncier et l'eau d'irrigation par le biais 
RECHERCHE

Guillaume SOULLIER, Paule MOUSTIER, Jérémy BOURGOIN, Alpha BA

Tableau 2. Liens entre les agrobusiness et les différents types de petits producteurs

\begin{tabular}{|c|c|c|c|}
\hline \multicolumn{4}{|c|}{ Les acteurs } \\
\hline Nom de l'AB & Senhuile-Senethanol & West African Farm & Coumba Nor Thiam \\
\hline Localisation & Ferlo & Diéri & Walo \\
\hline $\begin{array}{l}\text { Types de petits produc- } \\
\text { teurs }\end{array}$ & Agropasteurs & Producteurs diversifiés & Riziculteurs \\
\hline $\begin{array}{l}\text { Principales sources de } \\
\text { revenu des petits pro- } \\
\text { ducteurs }\end{array}$ & $\begin{array}{l}\text { Élevage, cultures } \\
\text { non irriguées }\end{array}$ & $\begin{array}{l}\text { Riz, maraîchage et } \\
\text { élevage }\end{array}$ & Riz \\
\hline Foncier utilisé & $\begin{array}{l}\text { Zones de parcours, } \\
\text { surfaces non-immatricu- } \\
\text { lées et non irriguées }\end{array}$ & \multicolumn{2}{|c|}{$\begin{array}{l}\text { Périmètres irrigués collectifs ou privés gérés collec- } \\
\text { tivement }\end{array}$} \\
\hline Ethnies & Peulh & Wolof et Maure & Wolof et Maure \\
\hline \multicolumn{4}{|c|}{ Liens entre les petits producteurs et les $A B$} \\
\hline Contrat de production & $0 \%$ & $0 \%$ & $76 \%$ \\
\hline Emploi salarié & $11.1 \%$ & $9.02 \%$ & $2 \%$ \\
\hline Location de terre & $18.18 \%$ & $0 \%$ & $2 \%$ \\
\hline Accaparement de terres & $16.16 \%$ & $1.5 \%$ & $3 \%$ \\
\hline Aucun lien & $54.54 \%$ & $89.48 \%$ & $17 \%$ \\
\hline
\end{tabular}

de la coordination verticale. L'entreprise Senhuile-Senethanol a été introduite dans le delta par le Gouvernement sénégalais, sans prendre en compte la gestion coutumière des ressources agricoles utilisées par les communautés d'agropasteurs qui vivent dans la réserve sylvo-pastorale déclassée. Les producteurs écartés des négociations cherchent à réintégrer la gouvernance territoriale par le biais de la mobilisation de la société civile et de l'occupation physique des espaces (Chia et al., 2008). L'entreprise West African Farm s'est inscrite dans la gouvernance territoriale par le biais de la négociation avec le conseil communal, en proposant des contreparties contractualisées au profit des producteurs et de la localité, dont un meilleur accès à l'eau d'irrigation. En pratique, la répartition des terres nouvellement irrigables a été source de conflits au sein de la communauté du fait de l'absence de règle d'attribution. De plus, les producteurs n'ont pas pu financer les aménagements secondaires nécessaires à la mise en culture.

\section{Effets sur les producteurs}

\section{Accès au foncier}

L'arrivée d'investisseurs à capitaux étrangers, soutenue par l'État sénégalais, a provoqué un changement d'échelle du foncier. Les cessions de terres se sont faites sur de plus larges surfaces, et pour des spéculations parfois vouées à l'exportation. Beaucoup d'affectations portent sur des terres sableuses, dévolues à l'élevage. Les investisseurs essaient de se conformer à la législation et reçoivent des titres d'affectations par les collectivités locales.

L'atelier participatif a mis en évidence que l'avancée du front agricole exacerbe les tensions entre agriculteurs et agropasteurs. La question de l'accès à l'eau, notamment au niveau du lac de Guiers, est source de conflits (Papazian et al., 2016). L'arrivée d'AB fait naître auprès des 
populations locales un sentiment d'accaparement des terres. Néanmoins, malgré les critiques envers les $A B$, les individus acceptent les investissements du fait des contreparties offertes. Ces contreparties portent sur la construction d'aménagements et d'infrastructures socioéconomiques de base, telles que des écoles, des postes de santé et des mosquées. Certains $\mathrm{AB}$ peuvent aussi réaliser des dons aux collectivités locales. La population accepte donc l'arrivée des $\mathrm{AB}$ du fait de leur capacité à se substituer aux politiques publiques.

\section{Revenus et sécurité alimentaire des riziculteurs}

Les modèles économétriques montrent que les contrats de commercialisation n'ont pas d'impact significatif sur les revenus car les petits producteurs engagés dans ces contrats utilisent les mêmes quantités et qualités d'intrants que ceux commercialisant par des transactions spot (Soullier et Moustier, 2018). Il résulte une qualité de paddy, un rendement et un prix de vente équivalents à ceux obtenus par les producteurs commercialisant par des transactions spot. Le contrat de commercialisation est une innovation organisationnelle qui vise à assurer le remboursement des crédits et les approvisionnements des riziers. Néanmoins, ce contrat réduit l'insécurité alimentaire car il atténue la saisonnalité des prix. En contre-saison 2014, le prix de vente par kilogramme dans un contrat de commercialisation a varié de 112,5 FCFA à 137,5 FCFA alors que le prix des transactions spots a varié de 83,3 FCFA à 150 FCFA. Les producteurs en plus grande insécurité alimentaire remboursent leur crédit durant les six semaines suivant la récolte, quand le prix de vente du contrat est supérieur à celui de la transaction spot. Les quantités conservées sont utilisées pour la consommation alimentaire du ménage.
Dans un contexte de risque de défaut de remboursement du crédit, la proximité géographique et relationnelle entre les producteurs et les riziers permet de réduire les coûts de transactions. Le contrat de production est souvent l'unique alternative de crédit pour les producteurs exclus de la banque nationale (Soullier et Moustier, 2018). Il engendre un effet positif sur le revenu de ces producteurs (dont le profit s'élève à $28,5 \mathrm{FCFA} / \mathrm{kg}$ ) lorsque ces derniers sont comparés aux producteurs ne pouvant pas cultiver de riz par manque de crédit. Cet upgrading a lieu par le biais de l'accès au crédit.

Néanmoins, les producteurs participant à un contrat de production ont des revenus moins importants que ceux des producteurs bénéficiant d'un crédit de la CNCAS et commercialisant par transaction spot (Soullier et Moustier, 2018). L'écart de profit est de 15,63 FCFA $/ \mathrm{kg}$, soit 38,81\%. Cette différence est due à un écart de prix de vente. En effet, le contrat de production pourrait inclure un taux d'intérêt implicite élevé, du fait de la structure oligopolistique du segment du marché du crédit offert aux producteurs endettés auprès de la banque. De plus, il inclut une prime d'assurance utilisée pour couvrir le défaut de remboursement de certains producteurs. Ces coûts implicites réduisent de 17,26 FCFA/ $\mathrm{kg}$ le prix de vente des producteurs contractualisés par rapport à celui des producteurs financés par la CNCAS.

\section{Pratiques agricoles}

Les pratiques agricoles recommandées par la SAED et celles mises en œuvre par les producteurs sont présentées dans le tableau 3. La SAED soutient une riziculture intensive. Elle encourage l'utilisation de semences améliorées et certifiées sur des périmètres irrigués. Elle appuie l'emploi d'engrais, qui sont le phosphate de diammonium (DAP) et l'urée, et d'herbicides de synthèse, qui sont le Propanyl, 
le Weedone et le Londax. La SAED incite aussi à la double culture (Diouf, 2013).

Les recommandations de la SAED sont relativement suivies. Les producteurs vendant par des transactions spot utilisent des engrais et herbicides de synthèse : $99,44 \mathrm{~kg} / \mathrm{ha}$ de DAP, $348,8 \mathrm{~kg} /$ ha d'urée, 6,221/ha de Propanyl, 1,391/ha de Weedone et $42,6 \mathrm{~g} / \mathrm{ha}$ de Londax. Ils utilisent les résidus de récolte comme fumure organique $(80,3 \%)$ et réalisent peu de labour $(1,31 \%)$.
La coordination verticale ne modifie pas la nature des intrants utilisés, et modifie peu les quantités utilisées. Les producteurs engagés dans un contrat de production utilisent des quantités de Propanyl et de Londax similaires à celles des producteurs vendant par des transactions spot. Ils ont aussi une même intensité culturale $(1,22)$, et utilisent dans la même proportion les résidus pour fumure organique $(88,8 \%)$.

Les AB utilisent aussi des intrants de même nature que ceux des producteurs familiaux. Toutefois, des différences

Tableau 3. Comparaison des intrants utilisés et des rendements par modes de gouvernance

\begin{tabular}{|c|c|c|c|c|}
\hline & $\begin{array}{l}\text { Recomman- } \\
\text { dations de la } \\
\text { SAED }\end{array}$ & $\begin{array}{l}\text { Transac- } \\
\text { tion spot }\end{array}$ & $\begin{array}{l}\text { Contrat de } \\
\text { production }\end{array}$ & $\begin{array}{l}\text { Hiérarchie } \\
\text { (AB) }\end{array}$ \\
\hline Nombre d'observations & & 61 & 27 & 30 \\
\hline Rendement (T/ha) & & 7,64 & $6,67 * * *$ & $6,94 * * *$ \\
\hline Réalisation d'un labour (\%) & Tous les 3 ans & $1,31 \%$ & $0,4 \% * * *$ & $0 \% * * *$ \\
\hline Semence (kg/ha) & $80-120$ & 128,24 & $145,15 * * *$ & $142,24 * * *$ \\
\hline $\begin{array}{l}\text { Parcelles semées hors période recom- } \\
\text { mandée }(\%)\end{array}$ & & $39 \%$ & $22 \%$ & $13 \% * * *$ \\
\hline $\begin{array}{l}\text { Exploitants utilisant de l'urée ou du } \\
\text { phosphate de diammonium (\%) }\end{array}$ & & $100 \%$ & $100 \%$ & $100 \%$ \\
\hline Phosphate de diammonium (kg/ha) & 100 & 99,44 & $102^{*}$ & $96,6^{*}$ \\
\hline Urée (kg/ha) & $250-300$ & 348,8 & $330,8 * * *$ & $335 * * *$ \\
\hline Fumure organique (résidus de récolte) & & $80,3 \%$ & $88,8 \%$ & $100 \% * * *$ \\
\hline $\begin{array}{l}\text { Exploitants utilisant du Propanyl, du } \\
\text { Weedone ou du Londax (\%) }\end{array}$ & & $100 \%$ & $100 \%$ & $100 \%$ \\
\hline Propanyl (I/ha) & 8 & 6,22 & 5,43 & $4,93 * *$ \\
\hline Weedone (l/ha) & 1 & 1,39 & $0,66 * * *$ & $0,13 * * *$ \\
\hline Londax (g/ha) & 100 & 42,6 & 40,74 & 46,66 \\
\hline Nombre de drainages & 5 & 4,96 & $3,14 * * *$ & $3,5 * * *$ \\
\hline Irrigation par groupe motopompe (\%) & & $6,56 \%$ & $14,81 \% * * *$ & $77,8 \% * * *$ \\
\hline Mauvaise qualité du planage (\%) & & $4,92 \%$ & $33,33 \% * * *$ & $51,85 \% * * *$ \\
\hline Mauvaise qualité du drainage (\%) & & $3,28 \%$ & $22,15 \% * * *$ & $40,74 \% * * *$ \\
\hline Intensité culturale & 2 & 1,15 & 1,22 & $1,95 * * *$ \\
\hline Taux de rotation (6 dernières saisons) (\%) & & $7,41 \%$ & $17,24 \%$ * & $0 \%$ * \\
\hline
\end{tabular}


existent sur les plans de l'intensité culturale et de la qualité des aménagements hydro-agricoles. L'intensité culturale des $\mathrm{AB}$ est supérieure $(1,95)$ à celle des agriculteurs familiaux $(1,15)$. En effet, l'action collective de ces derniers, relative à l'accès aux intrants, à l'irrigation et à la mécanisation, provoque des retards et entrave l'enchaînement de la contre-saison et de l'hivernage (Diagne et al., 2013). Les $\mathrm{AB}$ ont une plus grande autonomie, notamment par l'utilisation de groupes motopompes $(77,8 \%)$ sur des périmètres irrigués privés. De plus, les AB utilisent des quantités d'intrants légèrement inférieures à celles des producteurs vendant par des transactions spot : 96,6 kg/ha de DAP, $335 \mathrm{~kg} / \mathrm{ha}$ d'urée, 4,93 1/ha de Propanyl, et $0,131 /$ ha de Weedone. Cela peut être expliqué par une qualité différente des aménagements hydro-agricoles. Beaucoup de parcelles cultivées par des $\mathrm{AB}$ sont localisées sur des périmètres irrigués privés, et jugées de mauvaise qualité sur le plan du planage $(51,85 \%)$ et du drainage $(40,74 \%)$. Deux AB ont déclaré utiliser un peu moins d'intrants de synthèse pour limiter le risque économique.

La riziculture dans le delta est donc intensive, indépendamment des formes de gouvernance. Toutefois, les AB ont une plus forte intensité culturale et utilisent légèrement moins d'intrants de synthèse. Ces différences rendent difficile une anticipation des effets à moyen terme sur la reproduction de la fertilité des sols.

\section{Discussion}

Le croisement des cadres conceptuels de la gouvernance des chaînes de valeur et de la gouvernance territoriale met en évidence que le changement de gouvernance territoriale provoqué par les investissements d'AB dépend de la prise en compte des institutions coutumières et légales, et que les effets de la coordination verticale sont différents en fonction des types de producteurs (figure 3).

Les études de cas montrent que lors de l'arrivée d'un $\mathrm{AB}$ sur un territoire, la prise en compte des institutions coutumières et légales influence l'évolution de la gouvernance territoriale. L'entreprise Senhuile-Senethanol n'a pas consulté les petits producteurs et a mis en œuvre un mode de gouvernance territoriale basé sur la communication quant à ses décisions de gestion. La non-prise en compte des institutions coutumières de gestion du foncier et de l'eau d'abreuvement a mené à une mobilisation des petits producteurs visant à réintégrer la gouvernance territoriale. Le cas de West African Farm montre que la négociation d'un $\mathrm{AB}$ avec les producteurs familiaux ne suffit pas à la mise en œuvre d'une gouvernance territoriale qui leur est favorable. En effet, ces producteurs, représentés par le conseil rural, ont négocié des aménagements hydro-agricoles mais la règle d'attribution du foncier irrigué entre les producteurs n'a pas été clarifiée, et seule une partie des aménagements a été réalisée. Cela a été source de tensions au sein de la communauté. Toutefois, une partie des producteurs familiaux accepte l'arrivée des AB car ils fournissent certains biens publics. Pour que les petits producteurs ne voient pas leur contrôle des ressources agricoles territoriales diminuer, il semble nécessaire que l'implantation des $\mathrm{AB}$ prenne en compte les règles coutumières de gestion des ressources agricoles, en plus d'identifier des dispositifs de contrôle et d'application des accords établis.

D'autre part, le croisement des cadres conceptuels de la gouvernance des chaînes de valeur et de la gouvernance des territoires met en évidence que les investissements d'AB ont des effets qui diffèrent en fonction des types de producteurs. Les AB s'implantent dans des zones agroécologiques différentes, où des producteurs 
familiaux exercent des activités agricoles variées, ce qui donne lieu à des liens et à des effets différents.

Dans le cas des riziculteurs, localisés dans le Walo, les enquêtes montrent que la tendance vers l'intégration verticale provoque un upgrading. Le contrat de production a un effet positif sur le revenu des petits producteurs exclus du crédit de la CNCAS. Le contrat de commercialisation a un effet positif sur la sécurité alimentaire des petits riziculteurs. Il y a aussi un effet de l'intégration verticale sur le revenu des petits producteurs par le biais du salariat. Toutefois, cet upgrading est limité. Une partie de la littérature traite des impacts de la coordination verticale dans le cas de chaînes de valeur d'exportation de produits horticoles. Elle met en évidence que les contrats permettent l'accès à des intrants de synthèse et à des conseils techniques, ce qui provoque une amélioration des rendements, de la qualité du produit et du revenu (Reardon et al., 2009). Dans le cas de la chaîne de valeur du riz du delta du fleuve Sénégal, les contrats provoquent peu ou pas de modification des pratiques agricoles et des rendements puisque la riziculture a été intensifiée à partir de 1973. L'effet positif du contrat de production opère par le biais de l'accès au crédit pour les producteurs exclus de la banque nationale. Il engendre toutefois un profit par kilogramme inférieur à celui des riziculteurs financés par cette banque. En plus, le salariat pour un $\mathrm{AB}$ contrôlant hiérarchiquement la riziculture touche peu de ménages. Enfin, les effets de l'intégration verticale sur la fertilité des sols restent incertains puisque l'intensité culturale augmente mais les $\mathrm{AB}$ utilisent des quantités légèrement inférieures d'intrants de synthèse.

Pour les producteurs diversifiés, localisés dans le Diéri, les enquêtes montrent qu'il y a peu d'effets de l'intégration verticale. Le contrôle hiérarchique de la production influence positivement le revenu par le biais du salariat, mais peu de ménages sont concernés par une embauche. Ceci fut déjà constaté en Afrique et Amérique latine (Burnod et al., 2012). L'amélioration de l'accès au foncier ne concerne aussi qu'une petite partie des producteurs. L'AB ne s'approvisionne pas auprès des agriculteurs familiaux.

Pour les agropasteurs, les enquêtes montrent que les effets sont surtout négatifs. Les terres du Ferlo sont considérées comme non utilisées par les autorités centrales et locales. En effet, l'élevage extensif et les cultures pluviales dans cet espace n'ont pas été prévus comme une forme de mise en valeur par la loi sénégalaise. Ces terres sont néanmoins utilisées par les agropasteurs, en accord avec l'institution foncière coutumière. Une partie de cet espace a été attribuée à des $\mathrm{AB}$, dont les activités entrent en conflit avec les cultures pluviales et le pastoralisme. L'accaparement de foncier non immatriculé concerne un nombre important de producteurs. Les agropasteurs voient aussi leur accès à l'eau d'abreuvement contraint. Ces deux facteurs affectent négativement leur revenu et leur sécurité alimentaire. L'intégration verticale de la production agricole par les AB est créatrice d'emplois, mais cela concerne peu de ménages.

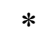

À la suite de la crise des prix de 2007, les États ouest-africains ont développé des politiques encourageant les investissements dans la production et la transformation agroalimentaire. L'arrivée d'AB sur des territoires où vivent des agriculteurs familiaux peut avoir des effets sur la gestion des ressources agricoles et la performance de ces petits producteurs. Nous utilisons les cadres théoriques de la gouvernance des chaînes de valeur (Gereffi et al., 2005) et de la gouvernance territoriale (Beuret, 
Figure 3. Chemins d'impacts entre les investissements d'agrobusiness et la performance des agriculteurs familiaux dans le delta de la vallée du fleuve Sénégal

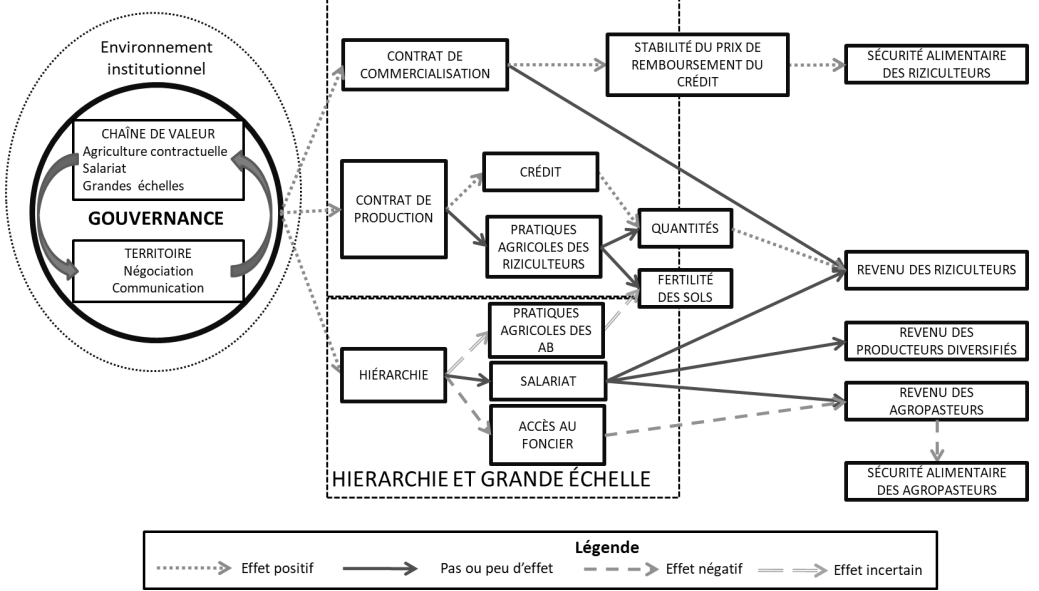

Source : les auteurs.

2006) pour comprendre quels sont les effets des investissements d'AB sur (1) la participation des petits producteurs dans la gestion des ressources agricoles du territoire, ainsi que sur (2) leur accès au foncier, revenu, sécurité alimentaire et pratiques agricoles. Pour répondre à ces questions, nous avons réalisé des entretiens semi-directifs, deux enquêtes quantitatives et un atelier participatif.

Le territoire étudié est le delta du fleuve Sénégal, où vivent 45000 petits producteurs. Les riziculteurs, agriculteurs diversifiés et agropasteurs occupent des zones agroécologiques différentes. En réponse à la crise des prix, les politiques agricoles ont encouragé les investissements, notamment par des attributions foncières. Des $A B$ ont investi dans la production et parfois la transformation de paddy, de produits maraîchers et d'agrocarburants. Ils mettent en place des stratégies différentes d'insertion territoriale. La tendance vers l'intégration verticale se manifeste par la mise en place de contrats et de relations salariales avec les exploitants familiaux.

Le croisement des cadres conceptuels de la gouvernance des chaînes de valeur et de la gouvernance territoriale met en évidence qu'avec les investissements d'AB, l'évolution de la participation des petits producteurs dans la gestion des ressources agricoles du territoire dépend de la prise en compte des institutions coutumières et légales. De plus, les effets de la coordination verticale sur les petits producteurs sont différents en fonction des types de producteurs. Les riziculteurs bénéficient de crédits et de contrats stabilisant leur prix de vente, alors que les agropasteurs voient une partie des espaces dédiés au pâturage et aux cultures non irriguées occupées par des investissements à grande échelle. Des relations salariales sont aussi observées, avec un effet positif sur le revenu, mais elles concernent un nombre limité de petits producteurs. Enfin, le contrôle hiérarchique de la production risque de limiter la reproduction de la fertilité des sols. 
De futures recherches pourraient continuer à croiser les cadres conceptuels des chaînes de valeur et des territoires afin de questionner les effets d'investissements d'agrobusiness en Afrique de l'Ouest. Elles pourraient particulièrement être appliquées aux pôles de croissance agricoles. Ce modèle, qui est promu par la Banque mondiale et la Banque africaine de développement, vise à ce que des investissements publics créent un contexte économique favorable aux investissements privés, souvent étrangers. Il est progressivement mis en œuvre en Côte d'Ivoire, au Burkina Faso, au Mali et dans d'autres pays de la région. Des recherches pourraient continuer à informer les politiques publiques des processus et effets des transformations liées aux pôles de croissance agricoles.

\section{RÉFÉRENCES BIBLIOGRAPHIQUES}

Actionaid (2014). Quel avenir sans ma terre? Des communautés mobilisées pour récupérer leur terre. Dakar, Actionaid. En ligne : https://www.farmlandgrab.org/ uploads/attachment/Quel_avenir_sans ma_terre.pdf (consulté le 21 août 2017).

Amanor K. (2012). Global resource grabs, agribusiness concentration and the smallholder: two west african case studies. The Journal of Peasant Studies, vol. 39, n 3-4, pp. 731-749. En ligne : https://doi.org/10. 1080/03066150.2012.676543 (consulté le 23 février 2017).

Bellemare M., Novak L. (2017). Contract farming and food security. American Journal of Agricultural Economics, vol. 99, $\mathrm{n}^{\circ}$ 2, pp. 357-378. En ligne : https://doi. org/10.1093/ajae/aaw053 (consulté le 20 juillet 2017).

Beuret J. (2006). La conduite de la concertation. Paris, L'Harmattan.

Burnod P., Colin J.-P., Anseeuw, W., Cheyns E., Clerc J., Faure G., Freguin Gresh S., Ruf F., Vagneron I., Vognan G. (2012). Grands investissements agricoles et inclusion des petits producteurs : leçons d'expériences dans 7 pays $d u$ Sud. Montpellier, Cirad.

Chia E., Torre A., Rey-Valette H. (2008). Conclusion : vers une «technologie » de la gouvernance territoriale! Plaidoyer pour un programme de recherche sur les instruments et dispositifs de la gouvernance des territoires. Norois, n 209, pp. 167-177. En ligne : https://doi.org/10.4000/norois.2603 (consulté le 17 December 2015].
Collectif de Ndiaël, CNCR, Enda Pronat, ActionAid Senegal, ActionAid Italy, Peuples Solidaires, Re:Common, Grain and Oakland Institute (2014). Des paysans et éleveurs sénégalais exigent que la multinationale Senhuile SA quitte leurs terres. En ligne : https://www.grain.org/fr/article/ entries/4900-des-paysans-et-eleveurs-senegalais-exigent-que-la-multinationale-senhuile-sa-quitte-leurs-terres (consulté le 9 décembre 2016).

Commune rurale de Ngnith (2011). Convention stratégique ente la société West African Farm et la communauté rurale de Ngnith. Ross Bethio, Commune rurale de Ngnith. En ligne : https:/www.farmlandgrab.org/ uploads/attachment/ContratWAF.pdf (consulté le 23 août 2017].

D’Aquino P., Seck S. M. (2013). Projet de développement inclusif et durable de l'agribusiness au Sénégal (PDIDAS). Développement de méthodes d'allocation de terrains par les communautés rurales et identification de leurs besoins en assistance technique. Zones de Gandon et du lac de Guiers. Centre de coopération Internationale en Recherche Agronomique pour le Développement. En ligne : https://agritrop.cirad.fr/570822/1/document_570822.pdf (consulté le 23 août 2017).

Diagne M., Demont M., Seck P., Diaw A. (2013). Self-sufficiency policy and irrigated rice productivity in the Senegal River Valley. Food Security, vol. 5, n ${ }^{\circ} 1$, pp. 55-68. En ligne : https://doi.org/10.1007/ s12571-012-0229-5 (consulté le 15 avril 2014). 
Diouf M. M. (2013). La commercialisation $d u$ riz local, doit-on aller vers le protectionnisme? Bulletin d'analyse économique Filière riz. Saint-Louis : Centre de Gestion et d'Économie rurale de la Vallée. En ligne : https://lavoixdelavallee.files.wordpress.com/2013/05/ae-com1-riz-nov-13.pdf (consulté le 23 août 2017).

Diouf M. M. (2014). La filière oignon $d u$ Sénégal. Bulletin d'analyse économique - Filière oignon, Saint-Louis, Centre de Gestion et d'Économie rurale de la Vallée. En ligne : https://avoixdelavallee.files. wordpress.com/2013/05/la-filic3a8re-oignon-au-sc3a9nc3a9gal.pdf (consulté le 23 août 2017).

Diouf M. M. (2016). La tomate industrielle du Sénégal: une filière en danger. Bulletin d'analyse économique - Filière tomate, Saint-Louis, Centre de Gestion et d'Économie rurale de la Vallée. En ligne : https://avoixdelavallee.files. wordpress.com/2017/01/la-filic3a8re-tomate-au-sc3a9nc3a9gal.pdf (consulté le 23 août 2017).

Fall A. A. (2006). Impact du crédit sur le revenu des riziculteurs de la Vallée du Fleuve Sénégal. Thèse de doctorat, Économie et gestion du développement agricole, agroalimentaire et rurale, Montpellier, École Nationale Supérieure d'Agronomie de Montpellier et Université Montpellier I. En ligne : http://www.bameinfopol.info/IMG/pdf/ These_AAFall.pdf (consulté le 23 août 2017).

Gereffi G., Humphrey J., Sturgeon T. (2005). The governance of global value chains. Review of International Political Economy, vol. 12, $\mathrm{n}^{\circ} 1$, pp. 78-104. En ligne : https:// doi.org/10.1080/09692290500049805 (consulté le 23 août 2017).

Gereffi G., Korzeniewicz M. (1994). Commodity chains and global capitalism. Contributions in economics and economic history. Westport, Conn: Greenwood Press.

Hathie I. (2016). Politiques d'autosuffisance en riz en Afrique de l'Ouest : quels acquis, quelles limites et quels débats? Bulletin de synthèse souveraineté alimentaire, Dakar, Initiative Prospective Agricole et Rurale. En ligne : http://www.inter-reseaux.org/IMG/ pdf/bds23_riz.pdf (consulté le 23 août 2017).
Humphrey J. (2004). Upgrading in global value chains. Working Paper. Geneva: International Labour Office. En ligne : https://papers.ssrn.com/sol3/papers.cfm?abstract_ $\mathrm{id}=908214$ (consulté le 24 août 2017).

Kamara S. (2014). Développements hydrauliques et gestion d'un hydrosystème largement anthropisé : le delta du fleuve Sénégal. Thèse de doctorat, Géographie, Avignon, Université d'Avignon et Université Gaston Berger. En ligne : https:/hal.inria. fr/file/index/docid/965106/filename/ThA_ se_Saliou-Kamara_Version-dA_finitive. pdf (consulté le 24 août 2017).

Lançon F., Mendez del Villar P. (2013). Le marché rizicole ouest-africain et la sécurité alimentaire: leçons et perspectives après la flambée des prix de 2008. Paris, Club Demeter. En ligne : http://agritrop. cirad.fr/570490/1/document_570490.pdf (consulté le 24 août 2017).

MA (2009). Programme national d'autosuffisance en riz. Rapport intermédiaire. Dakar, Ministère de l'Agriculture de la République du Sénégal. En ligne : http://www.ipar.sn/ IMG/pdf/2009_Prog_Nat_Autosufisance_ Riz.pdf (consulté le 24 janvier 2017).

Medernach K., Burnod P. (2013). Recompositions inattendues d'un système agraire malgache par l'agrobusiness. Études rurales, vol. 1, $\mathrm{n}^{\circ} 191$, pp. 63-76. En ligne : https:/etudesrurales.revues. org/9777 (consulté le 24 août 2017).

Opondo M. (2000). The socio-economic and ecological impacts of the agro-industrial food chain on the rural economy in Kenya. Ambio, vol. 29, $\mathrm{n}^{\circ}$ 1, pp. 35-41. En ligne : http://dx.doi.org/10.1579/0044-7447-29.1.35 (consulté le 17 novembre 2016).

Papazian H., D’Aquino P., Bourgoin J., Ba A. (2016). Jouer avec diverses sources de régulation foncière : le pluralisme sahélien. Économie rurale, $\mathrm{n}^{\circ}$ 353-354., pp. 27-44. En ligne : https://doi.org/10.4000/economierurale.4904 (consulté le 25 janvier 2017).

Reardon T., Barrett C., Berdegué J., Swinnen J. (2009). Agrifood industry transformation and small farmers in developing countries. World Development, vol. 37, $\mathrm{n}^{\circ} 11$, pp. 17171727. En ligne : https://doi.org/10.1016/j.worlddev.2008.08.023 (consulté le 3 mai 2013). 
Rey-Valette H., Pinto M., Maurel P., Chia E., Guihéneuf P.-Y., Michel L., Nougaredes B., Soulard C., Jarrige F., Guillemot S., Cunnac M., Mathé S., Barbe E., Ollagnon M., Delorme G., Prouhèze N., Laurent A., Suita L., Bertrand J., Dieudonné C., Morvan R., Champrigaud A. (2011). Guide pour la mise en cuvre de la gouvernance en appui au développement durable des territoires. Projet PSDR GouvInnov, Languedoc-Roussillon, Série Les focus PSDR3. Montpellier. En ligne : http://agris.fao.org/agris-search/search. do?recordID=FR2014010652 (consulté le 17 décembre 2015).

Soullier G. (2017). Modernization of domestic food chains in developing countries: what effects on small-scale farmers? The rice value chain in Senegal. Thèse de doctorat, sciences économiques. Montpellier, Université de Montpellier.

Soullier G., Moustier P. (2015). Does modernization of the rice value chains in Senegal illustrate a move toward the asian quiet revolution? 9th seminar of research in social sciences, December 10-11, 2015, Nancy (Université de Lorraine). En ligne : https://afrique-ouest.cirad.fr/content/download/6641/61901/version/1/file/obj 5614 file_Soullier-et-Moustier.pdf and http:// ageconsearch.umn.edu/record/245704 (consulté le 23 août 2017).

Soullier G., Moustier P. (2018). Impacts of contract farming in domestic grain chains on farmer income and food insecurity. Contrasted evidence from Senegal. Food Policy., vol.79.pp. 179-198. En ligne: https:// doi.org/10.1016/j.foodpol.2018.07.004.

Soullier G., Moustier P., Ba A., Bourgoin J. (2016). Effets des agrobusiness sur les petits producteurs dans la vallée du fleuve Sénégal. Montpellier, Cirad.

Van den Broeck G., Swinnen J., Maertens M. (2017). Global value chains, large-scale farming, and poverty: long-term effects in Senegal. Food Policy, vol. 66, pp. 97-107. En ligne : https://doi.org/10.1016/j.foodpol.2016.12.003 (consulté le 9 janvier 2017). 